\title{
Correction to: Purging behaviors relate to impaired subjective sleep quality in female patients with anorexia nervosa: a prospective observational study
}

Tokusei Tanahashi ${ }^{1,2^{*}}$, Keisuke Kawai ${ }^{1}$, Keita Tatsushima ${ }^{1}$, Chihiro Saeki ${ }^{1}$, Kunie Wakabayashi ${ }^{1}$, Naho Tamura ${ }^{1}$, Tetsuya Ando ${ }^{3}$ and Toshio Ishikawa ${ }^{1}$

\section{Correction}

After publication of the original article [1], the authors reported an error to Table 2.

The title for the right-hand column of Table 2 was incorrectly included as " $\rho$ " when it should have been "P". The correct version of Table 2 is included in this Correction.

Table 2 Spearman's rank correlation analysis of global sleep quality (global PSQI-J)

\begin{tabular}{lll}
\hline Variables & $\rho$ & $P$ \\
\hline Diagnosis of ANbp & 0.525 & $0.017^{*}$ \\
Age & 0.345 & 0.136 \\
Body mass index & 0.168 & 0.479 \\
Duration of illness & 0.536 & $0.015^{*}$ \\
Current menstruation & 0.281 & 0.230 \\
Use of sedative drugs & 0.332 & 0.152 \\
CES-D & 0.318 & 0.172 \\
Vomiting & 0.561 & $0.010^{*}$ \\
Chewing & -0.061 & 0.798 \\
Laxative overuse & 0.407 & 0.075 \\
Uretic misuse & 0.280 & 0.232 \\
\hline
\end{tabular}

ANbp anorexia nervosa binge-eating/purging type; CES-D Center for Epidemiologic Studies Depression, PSQI-J Japanese version of the Pittsburgh Sleep Quality Index; $\rho$ Spearman's correlation coefficient

${ }^{*} P<0.05$

\section{Author details}

${ }^{1}$ Department of Psychosomatic Medicine, Kohnodai Hospital, National Center for Global Health and Medicine, 1-7-1 Kohnodai, Ichikawa, Chiba 272-8516, Japan. ${ }^{2}$ Department of Psychosomatic Medicine, Saiseikai Fukuoka General Hospital, 1-3-46 Tenjin, Chuo-ku, Fukuoka 810-0001, Japan. ${ }^{3}$ Department of Psychosomatic Research, National Institute of Mental Health, National Center of Neurology and Psychiatry, 4-1-1 Ogawahigashi, Kodaira, Tokyo 187-8553, Japan.

Received: 26 February 2018 Accepted: 26 February 2018 Published online: 15 March 2018

\section{Reference}

1. Tanahashi T, Kawai K, Tatsushima K, Saeki C, Wakabayashi K, Tamura N, et al. Purging behaviors relate to impaired subjective sleep quality in female patients with anorexia nervosa: a prospective observational study. BioPsychoSocial Medicine. 2017;11:22.

\footnotetext{
* Correspondence: tanatok@med.kyushu-u.ac.jp

'Department of Psychosomatic Medicine, Kohnodai Hospital, National Center for Global Health and Medicine, 1-7-1 Kohnodai, Ichikawa, Chiba 272-8516, Japan

${ }^{2}$ Department of Psychosomatic Medicine, Saiseikai Fukuoka General Hospital, 1-3-46 Tenjin, Chuo-ku, Fukuoka 810-0001, Japan
} 